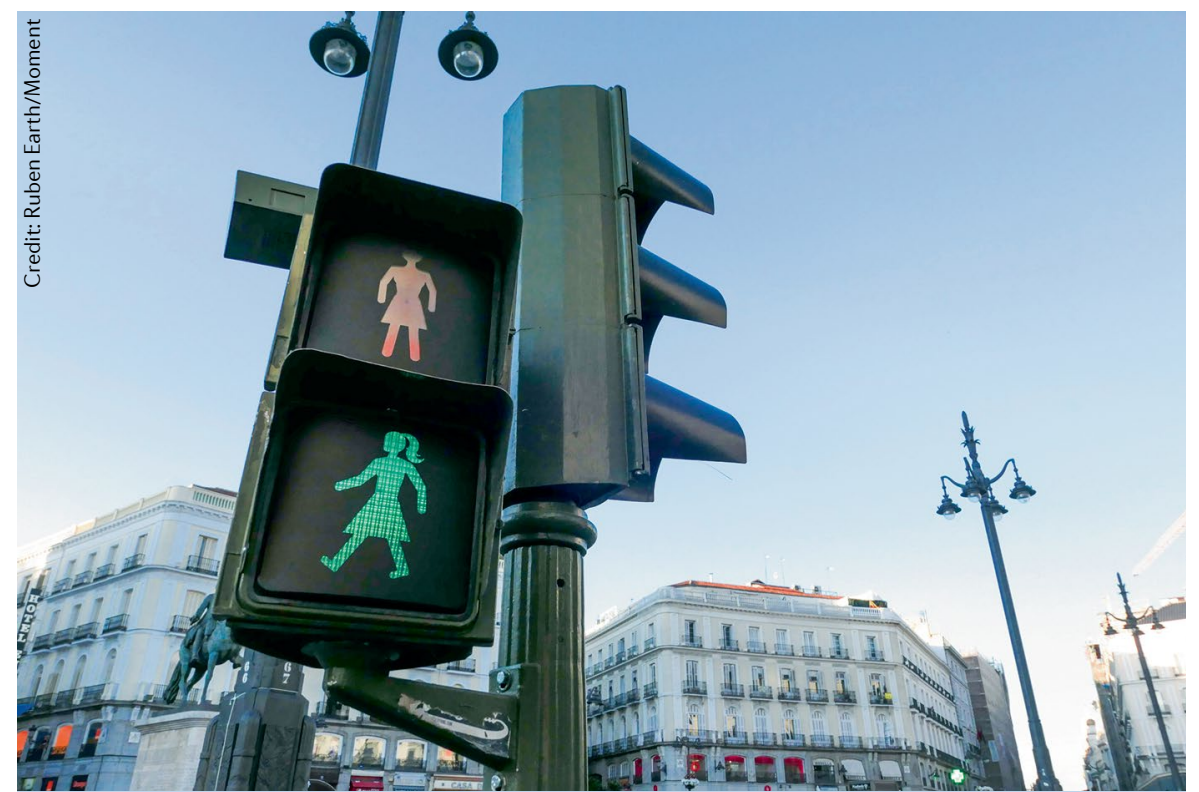

ע) SCIENCE AND SOCIETY

\title{
The road to equity for women in academic rheumatology
}

\section{Shereen N. Mahmood and Irene Blanco}

\section{Gender disparities persist in many aspects of working life for women in} academic rheumatology. To move forward, we must find ways to address the gender gap in rheumatology with the goal of creating a workforce as diverse as the patient population it serves.

Refers to Jorge, A. et al. The association between physician gender and career advancement among academic rheumatologists in the United States. Arthritis Rheumatol. https://doi.org/10.1002/art.41492 (2020) | Bagga, E. et al. Representation of women as authors of rheumatology research articles. Arthritis Rheumatol. https://doi.org/10.1002/art.41490 (2020).

Two articles published in Arthritis \& Rheumatology have highlighted the persistent gender disparities in promotion, publication and federal funding for women in academic rheumatology in the USA ${ }^{1,2}$. These discrepancies persist in spite of parity in leadership positions. These articles bring into question the many challenges that are still faced by women in achieving gender equity in academic careers; in this commentary, we specifically consider the role of academic tracks and research-specific inequities.

In their brief report, Jorge et al. analysed data from a 2014 cohort of rheumatologists derived from an online database of physicians in the USA with a valid national provider identifier ${ }^{1}$. They found that women in this cohort were less likely to have been promoted to the rank of associate or full professor, had fewer publications and were awarded fewer federal grants than men. However, when they looked at the likelihood of holding leadership positions, women were just as likely as men to have the title of programme director or division director. As acknowledged by Jorge et al. ${ }^{1}$, women are predicted to comprise the majority of the rheumatology workforce by $2025\left(\right.$ REF. $\left.^{3}\right)$. Therefore, although it is women face gender bias in publishing and funding mechanisms, thus potentially stalling promotion

heartening that women have achieved parity in holding leadership roles within their divisions, it is concerning that they continue to lag behind men in several other important academic metrics.

In their analysis of the nature of gender disparities in academic rheumatology, one thing that Jorge et al. did not investigate (but is important to note) was the promotion track that an academic rheumatologist might find herself on. Women in academic medical centres in the USA tend to be on clinicianeducator tracks ${ }^{4}$, whereas Jorge et al. used metrics that are predominantly associated with traditional, research-focused tenure tracks to analyse the career advancement of the rheumatology workforce ${ }^{1}$. Although it is crucially important that women are adequately represented in research careers, it could be that the majority of women in rheumatology are not on traditional tenure tracks, and as such, their path to career advancement will be different. This fact is especially important given that women on clinicianeducator tracks are at a higher risk of leaving academia than women on research tracks ${ }^{4,5}$. As noted in a 2006 review article ${ }^{6}$, the role of the clinician-educator is crucial for the education of resident physicians and especially for rheumatology fellows; however, given that the metrics for promotion often rely on the publication of basic, clinical and translational research, clinician-educators often lag behind their peers in other tracks. As the author of the review recommends, promotion tracks should be modified, and clinician-educators should be championed and provided with sufficient faculty development to not only become master educators, but also scholars in medical education.

The finding that women are just as likely to have leadership roles as men in the cohort studied by Jorge et al. ${ }^{1}$ is incredibly encouraging. As we investigate this finding further, we need to ensure that there is equity in these roles and that women who are programme or division directors are supported, compensated and sponsored in the same way that men 
would be in the same positions. Although this fine detail has not been fully studied in rheumatology, lessons can be learnt from academic cardiology, where an increasing proportion of women are programme directors and division directors ${ }^{7}$. Despite this increase, on average, cardiology programme directors who are men were of higher rank, had more publications in total and had publications in higher impact journals than those who are women. This discrepancy was not true for cardiology division directors, as women seem to have achieved parity with men in the same metrics ${ }^{7}$. Again, given that programme directors have a predominantly educational role, it behoves deans, department chairs and division chiefs to evaluate how education is valued on the track for promotion, especially if we are to retain women in academia.

\section{we must also continue to} consider how else we can diversify the field to provide
long-term equity

The majority of women might be on clinician-educator tracks in academic rheumatology; however, we must also acknowledge the experience of women on research-focused tracks. Bagga et al. conducted a study looking at the percentage of women in lead and senior author positions in articles that report rheumatology-related research ${ }^{2}$. Overall, they found gender parity in first authorship for publications reporting investigator-lead research. However, despite this finding, there were still fewer women than men listed as senior authors, and women were under-represented as first and last authors for publications reporting industry-sponsored studies and randomized controlled trials. Although it is encouraging that women are well represented as lead authors, the reason for the lack of women in senior authorship positions in clinical trials and industry-led studies is unclear. This discrepancy might reflect the relative lack of women in advanced research careers. As noted by Bagga et al. ${ }^{2}$, women face gender bias in publishing and funding mechanisms, thus potentially stalling promotion along research tracks. In addition, it remains to be seen how the COVID-19 pandemic will affect female investigators and leaders within rheumatology. Not only are women publishing fewer articles than expected during this time, but they are also shouldering increased burdens secondary to the pandemic, including increased patient care, intensified work-life imbalance and sacrificed positional duties and title advancements ${ }^{8}$.

Lastly, we must acknowledge the intersectional experience of women in academia. Women of colour with rheumatic diseases, especially those with connective tissue diseases, have a high burden of disease activity and are also disproportionately affected by health disparities. Nevertheless, rheumatology has a workforce that, like much of medicine, does not reflect those patients who are most affected. In the 2015 ACR workforce study, few adult rheumatologists identified as Hispanic/Latinx (8.5\%), Black (0.8\%) American Indian/Alaska Native $(0.3 \%)$ or Native Hawaiian/Pacific Islander (0.1\%), and there were comparable numbers in paediatric rheumatology ${ }^{9}$. The proportion of these rheumatologists that are women is unknown. As we think about how to address gender equity in rheumatology, we must also continue to consider how else we can diversify the field to provide long-term equity for patients, practitioners and investigators.

As the future of rheumatology unfolds, and as we strive for excellence in patient care, research and education, we need to address the barriers that lead to inequities faced by patients and practitioners. The studies by Jorge et al. ${ }^{1}$ and Bagga et al. ${ }^{2}$ highlight a gender disparity in academic rheumatology that persists despite having been under discussion for many years ${ }^{10}$. Although major gains have been made in the number of women in rheumatology, further work must address the possible causes of inequity between women and men to ensure that women are not only adequately represented and advocated for, but also retained within academic divisions.

Shereen N. Mahmood $\bowtie$ and Irene Blanco iD $\bowtie$ Albert Einstein College of Medicine, Division of Rheumatology, Bronx, NY, USA.

凶e-mail:shmahmoo@montefiore.org; irene.blanco@einsteinmed.org

https://doi.org/10.1038/s41584-020-00517-7

1. Jorge, A. et al. The association between physician gender and career advancement among academic rheumatologists in the United States. Arthritis Rheumatol. https://doi.org/10.1002/art.41492 (2020).

2. Bagga, E. et al. Representation of women as authors of rheumatology research articles. Arthritis Rheumatol. https://doi.org/10.1002/art.41490 (2020).

3. Battafarano, D. F. et al. 2015 American College of Rheumatology Workforce Study: Supply and demand projections of adult rheumatology workforce, 2015-2030. Arthritis Care Res. 70, 617-626 (2018).

4. Mayer, A. P. et al. Gender distribution of U.S. medical school faculty by academic track type. Acad. Med. $\mathbf{8 9}$ 312-317 (2014).

5. Speck, R. M. et al. Factors impacting the departure rates of female and male junior medical school faculty: evidence from a longitudinal analysis. J. Womens Health 21, 1059-1065 (2012).

6. Passo, M. H. The role of the clinician educator in rheumatology. Curr. Rheumatol. Rep. 8, 469-473 (2006).

7. Khan, M. S. et al. Women in leadership positions in academic cardiology: A study of program directors and division chiefs. J. Womens Health 28, 225-232 (2019).

8. Brubaker, L. Women physicians and the COVID-19 pandemic. JAMA https://doi.org/10.1001/ jama.2020.14797 (2020).

9. American College of Rheumatology. 2015 Workforce Study of Rheumatology Specialists in the United States https://www.rheumatology.org/portals/0/files/ ACR-Workforce-Study-2015.pdf (ACR, 2017).

10. Lundberg, I. E. et al. Women in academic rheumatology. Arthritis Rheum. 52, 697-706 (2005).

Acknowledgements

The authors acknowledge the Association of Women in Rheumatology and all of the female mentors that they have had over the years who have paved the way for them.

\section{Competing interests}

The authors declare no competing interests. 